\title{
Bivariate Multilevel Modeling of Modern Contraceptive Use and Knowledge of HIV/AIDS Prevention of Women in Ethiopia
}

Daniel Bekele ( $\square$ daniadvent12@gmail.com )

Dire Dawa University https://orcid.org/0000-0003-0092-3756

Essey Kebede

\section{Research}

Keywords: modern contraceptive use, knowledge HIV, multilevel, bivariate multilevel

Posted Date: May 26th, 2021

DOI: https://doi.org/10.21203/rs.3.rs-524447/v1

License: (c) (i) This work is licensed under a Creative Commons Attribution 4.0 International License. Read Full License 
Bivariate Multilevel Modeling of Modern Contraceptive Use and Knowledge of HIV/AIDS Prevention of Women in Ethiopia.

Daniel Bekele ${ }^{1 *}$ Essey Kebede ${ }^{2}$

\section{Affiliations}

${ }^{1 *}$ Departments of Statistics, College of Natural and Computational Sciences, Dire Dawa University, PO Box: 1362, Dire Dawa, Ethiopia. Email: daniadvent12@gmail.com

${ }^{2}$ Departments of Statistics, College of Science, Bahir Dar University, Bahir Dar, Ethiopia Email: esseykebede@gmail.com

Correspondence author: daniadvent12@gmail.com

Departments of Statistics, College of Natural and Computational Sciences, Dire Dawa University, PO Box: 1362, Dire Dawa, Ethiopia. 


\begin{abstract}
Background: Ethiopia is one of the most populous countries in Africa and has the highest number of new HIV infections. Therefore, the main objectives of this study were to demonstrate the joint predictors and association between modern contraceptive use and knowledge of HIV prevention in women aged 15-49 across clusters in Ethiopia.
\end{abstract}

Methods: This study considered a weighted sample of 10,223 married women aged 15-49 years, extracted from the 2016 EDHS data. The bivariate multilevel logistics regression model was used in this study.

Results: In Ethiopia, $64.75 \%, 54.58 \%$ of the women didn't use the modern contraceptive method and didn't know about HIV prevention, respectively. A positive relationship between the use of modern contraceptives and knowledge of HIV prevention of women across the clusters was found, which implies that modern contraceptive use has positive implications on women's knowledge of HIV prevention techniques across the clusters.

Conclusions: Predictor visiting a health facility, wealth index, media exposure, religion, place of residence, and the contextual region are statistically related with the use of modern contraceptive use and knowledge of HIV prevention of women across the clusters. Those women who use the modern contraceptive method have more likelihood of knowing about HIV prevention. The study result confirmed that modern contraceptive use has a spillover effect on women's knowledge of HIV prevention across the clusters. Therefore, the integration of the two programs with taking into account their joint predictors was an advantage in terms of clinical benefit, cost, and for seeking care individuals in the communities. The integration of two programs with due consideration of community-specific was recommended.

Keywords: modern contraceptive use, knowledge HIV, multilevel, bivariate multilevel

\title{
Background
}

In the world in 2017, around 810 women die every day from the complication of pregnancy and childbirth-related problems and $94 \%$ of maternal mortality occurs in low- and lower-middle- 
income countries(Report, 2019; WHO, UNICEF, UNFPA, 2019). Women in developing countries have, on average many more pregnancies than women in developed countries, and also their lifetime risk of death due to the complication of pregnancy or childbirth is higher. Women living in developed countries have a maternal risk of death in which the probability that a 15-year-old woman will die of pregnancy complication is 1 in 6500 in Europe and 1in 7800 in Australia and New Zealand versus 1 in 37 in sub-Saharan Africa (Nations, 2019; WHO, UNICEF, UNFPA, 2019).

Most of the sexually active women of reproductive age 15-49 in developing countries and, in particular, Sub-Saharan Africa wanted to postpone pregnancy and delay childbearing to limit their family size, but they have an unmet need for family planning (Darroch, Sedgh and Ball, 2011). In Ethiopia, maternal mortality was 412 deaths per 100,000 live births and this death was mainly from the complication of unintended pregnancy. The 2016 EDHS report shows that only $35 \%$ of women aged 15-49 years are currently using modern contraceptives methods. Thus, the enhancement of family planning among women has a great role in improving the health of women, children, and families(ICF, 2016).

According to the Ethiopian Public Health Institute projection done in 2017, currently, a total of 433,666 adult women living with HIV infection and 12,307 adult women are caught with new HIV infection and 4,329 adult women have died from HIV/AIDS infection annually (Institute, 2017). Ethiopia has a plan to reduce new adult HIV infections by $50 \%$ and diminish the HIV/AIDS public threat by 2030(Federal HIV/AIDS Prevention and Control Office, 2020).

A study conducted on the use of modern contraceptive methods and uptake of HIV testing among women in Sub-Saharan Africa showed that women using modern contraceptive methods are more likely to be tested for HIV/AIDS diseases as compared to women not using a modern contraceptive method. The researchers also recommend that further studies should be conducted to clarify the relationships between the knowledge of HIV prevention and using modern contraceptives methods among women (Center et al., 2016). A study conducted in Sub-Saharan Africa on health system integrations of sexual and reproductive health and HIV services revealed that the integration of sexual reproductive health and HIV service is supported by major international health policies and donors(Hope et al., 2014). 
In Ethiopia, many scholars have conducted studies on the determinants of using modern contraceptive methods and knowledge of HIV/AIDS prevention separately (Girum et al., 2018; Gebre and Edossa, 2020; Hrusa et al., 2020). From the EDHS data, both information's about using or not using modern contraceptive methods and did know or did not know about HIV prevention was collected from the same women's across the clusters in Ethiopia and women living in the same community or clusters or EA share similar socio-economic and environmental factors. Therefore, the separate modeling of these outcomes has its drawback in providing a consistent estimate of the population parameter and valid statistical inference (LEONARDO et al., 2016; Yu et al., 2018). Therefore, this study was aimed to address these statistical gaps by taking into account the interdependence of women living in the same clusters in Ethiopia.

However, as far as the best of the researcher's knowledge, no study has been conducted on the joint predictors of modern contraceptive use and knowledge of HIV prevention by taking into account the interdependence between pairs of women in the same clusters in Ethiopia. Therefore, the current study aimed to fill these gaps and put a direction in which the two programs integrated in the future and gives an efficient health service for the community and to elaborate the relationship between modern contraceptive use and knowledge of HIV prevention in women across clusters by modeling the correlation between pairs of women in the same clusters by introducing unobservable random effects into the model.

\section{Methods}

\section{Data source, sampling design, and setting}

The datasets used in this study were taken from the 2016 EDHS. It was the fourth comprehensive and nationally representative survey conducted as the part of worldwide demographic and health survey(DHS) project. The 2016 EDHS samples were selected using a stratified two-stage cluster sampling design. In the first stage, around 645 enumeration areas (EAs) (202 in urban areas and 443 in rural areas ) were selected with probability proportional to EAs size and with independent selection in each stratum. The 2016 EDHS data were downloaded after getting authorization from the DHS website(https://dhsprogram.com ). The data collection was conducted from January 18, 2016, to June 27,2016 , which was designed to obtain and provide timely information on a key 
indicator of health and socio-demographic variable interest of the population (ICF, 2016). The study population was all reproductive women age 15-49 years in the selected EAs was eligible.

\section{Study variables}

In this study, two outcomes were considered. The first outcome was modern contraceptive use, which is categorized into using modern contraceptive (coded as 1) and not using modern contraceptive (coded as 0 ). Using modern contraceptive method was defined for those woman utilizing one these products such as female sterilization, male sterilization, the intrauterine contraceptive device, implants, injectable, the pill, male condoms, female condoms, emergency contraception, standard days method, and lactation amenorrhea method at the time of data collection, vise versa (ICF, 2016). The second outcome knowing HIV/AIDS prevention was categorized into know about HIV prevention (coded as 1) and do not know about HIV prevention (coded as 0). Knowing HIV prevention was defined as if the woman knows that using condoms and limiting the number of sexual partners to one uninfected partner at the time of data collection, vise versa (ICF, 2016).

The 2016 EDHS data have a hierarchal structure and in this study, women were considered level one and clusters as level-two(ICF, 2016). However, in this study three-level data structure was used with consideration of two responses as level-one, women as level-two, and clusters as levelthree as presented in

Figure 1. The lowest level of the data existed solely to define bivariate response structures of the data (Hox, 2010).

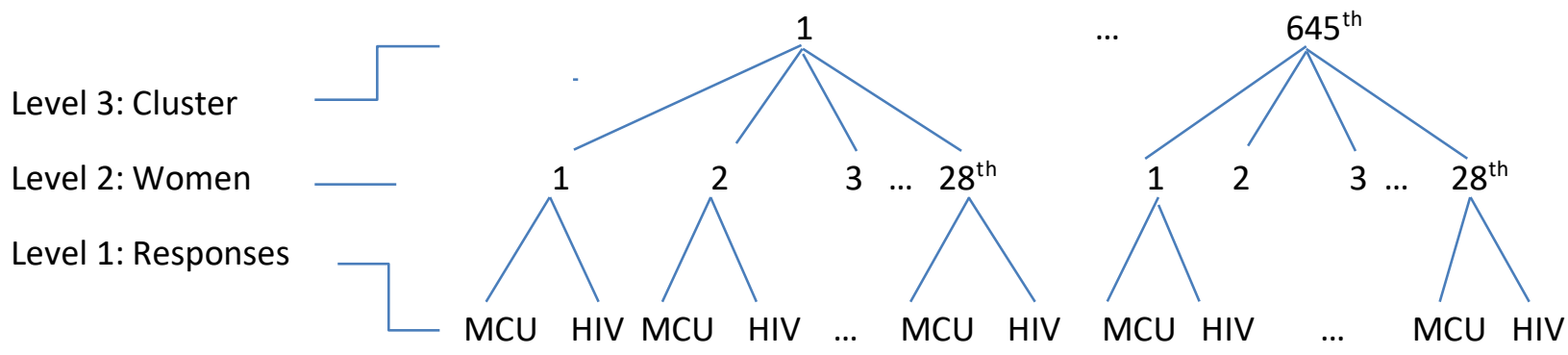

where MCU is modern contraceptive use. HIV is knowledge of HIV.

Figure 1: Hierarchal structures of the data 
In this study, the covariates proposed to be related to the use of modern contraceptive methods and knowledge of HIV/AIDS prevention were recruited based on the existing literature review as presented in Table 1.

Table 1: Independent variables and their description.

\begin{tabular}{ll}
\hline Variables & Codes \\
\hline Individual-level variable (level-1) & \\
Age of women & $0=15-24$ years, $1=25-34$ years, $2=35-49$ years \\
Education & $1=$ Higher, $2=$ No education, $3=$ Primary, $4=$ Secondary \\
Visited health facility last 12 month & $1=$ Yes, $0=$ No \\
Wealth index & $1=$ Richest, $2=$ Middle ,3= Poorer, 4=Poorest, 5= Richer \\
Distance to a health center & $1=$ Not big problem, $0=$ Big problem \\
Media exposure & $1=$ Yes , $0=$ No \\
Religious & $1=$ Orthodox, 2= Catholic, 3= Muslim,4= Protestant, 5= \\
& Other \\
\hline Community-level variable (level-2) & \\
\hline Place of residence & $1=$ Rural , $0=$ Urban \\
Contextual region & $1=$ City, $2=$ Pastoralist, $3=$ Agrarian \\
\hline
\end{tabular}

The contextual variables represent collective social characteristics of the group and in this study, the contextual region was categorized into city, pastoralist, and agrarian which is created from nine regions and two city administration with consideration of Addis Abeba, Dire Dawa, and Harar as the City contextual region: Somali and Afar as the Pastoralist contextual region: Tigray, Amhara, Oromia, SNNP, Gambela, and Benshangul Gumuz as the Agrarian contextual region agree with the study (Ousman, Magnus and Sundby, 2020).

\section{Statistical Analysis}

The analysis was done using SAS 9.4 statistical software. In this study, descriptive statistics such as cross-tabulation, Chi-square test of an association were employed to explore the relationships between two-response and predictor variables. Inferential statistics bivariate multilevel logistics regression was employed to examine the association between the two outcomes. A bivariate 
multilevel logistics regression model was the subsets of a generalized linear mixed model (GLMM) was applied to take into account the correlation of responses on modern contraceptive use and knowledge of HIV prevention of woman living in the same cluster. The generalized linear mixed model (GLMM) is an extension of the generalized linear model (GLM) by incorporating unobservable random effects as the linear predictor and linear mixed model (LMM) of continuous response as the special case (Breslow and Clayton, 1993). GLMM were used to account for unobservable factors in the dependent variables through individual-specific or cluster-specific in different levels of the data (Wang, 2013). Let us introduce superscript $r$ to enable us to distinguish the responses, for modern contraceptive use $\left(y_{i j}^{1}, r=1\right)$ and knowledge of HIV prevention $\left(y_{i j}^{2}, r=2\right)$ of a women $i$ in the community or EA. As in the separate modeling, in the bivariate model, each outcome follows the binomial distribution $Y_{i j}^{r} \sim \operatorname{bin}\left(1, \mu_{i j}^{r}\right)$ with conditional variance $\operatorname{var}\left(y_{i j}^{r} \mid \mu_{i j}^{r}\right)=\mu_{i j}^{r}\left(1-\mu_{i j}^{r}\right)$.

A bivariate multilevel logistics regression model with $P$ level one explanatory variable $x_{1}, \ldots, x_{P}$ and $Q$ level two explanatory variables $z_{1}, \ldots, z_{Q}$ has the following general form (Damon and Robert, 2015).

$$
\operatorname{logit}\left(y_{i j}^{r} \mid u_{i j}^{r}\right)=\beta_{o o}^{r}+\sum_{p=1}^{P} \beta_{p o}^{r} x_{p i j}^{r}+\sum_{q=1}^{Q} \beta_{q o}^{r} z_{q j}^{r}+u_{o j}^{r}
$$

where $y_{i j}^{r}$ represent $r^{t h}$ the responses of women $i$ in community $j$ where $r=1,2$. The term $u_{o j}^{r}$ represent random effects of community-specific difference for the $r^{\text {th }}$ responses.

where $u_{o j}^{r}$ - random-effects assumed to distributed multivariate normal as below.

$$
\left[\begin{array}{l}
u_{o j}^{1} \\
u_{o j}^{2}
\end{array}\right] \sim \operatorname{MVN}\left(0,\left[\begin{array}{cc}
\sigma_{u 1}^{2} & \sigma_{u 12} \\
\sigma_{u 12} & \sigma_{u 2}^{2}
\end{array}\right]\right)
$$

where the random effects for the two outcomes as assumed to be normally distributed with mean zero and $\Omega_{G}$ variance-covariance matrix. In a generalized linear mixed model, there are no exact suggested ways to evaluate the goodness fit of the model. However, the appropriate one is checking whether or not over or under dispersion in the data by using Pearson Chi-square over the degree 
of freedom, then no presence of over or under dispersion in the data due to the inclusion of randomeffects in the model was confirmed, and model goodness fit of the data to the model was adopted as presented in Table 3 (Schabenberger, 2005).

\section{Results}

\section{Socio-demographic characteristics of study participants}

A total of 10,223 married women aged (15-49 years) from 645 clusters was considered, results (Table 2) indicates that only about $35.25 \%$ of them use modern contraceptives and the majority $64.75 \%$ of married women were nonusers of modern contraceptive methods. Similarly, $45.42 \%$ of married women knew about HIV/AIDS prevention and the majority of $54.58 \%$ of married women were not know HIV/AIDS prevention methods across the clusters in Ethiopia.

Table 2: The bivariate analysis of modern contraceptive use and knowledge of HIV prevention of women across the clusters with their socio-economic and demographic predictors (EDHS 2016).

\begin{tabular}{|c|c|c|c|c|c|c|}
\hline \multirow[b]{2}{*}{ Variables } & \multicolumn{2}{|c|}{ Modern contraceptive use } & \multirow[b]{2}{*}{$\begin{array}{c}\text { Chi- } \\
\text { square } \\
\text { P-value }\end{array}$} & \multicolumn{2}{|c|}{ HIV prevention knowledge } & \multirow[b]{2}{*}{$\begin{array}{l}\text { Chi- } \\
\text { square } \\
\text { P-value }\end{array}$} \\
\hline & $\begin{array}{c}\text { Yes }(\%) \\
3604(35.25 \%)\end{array}$ & $\begin{array}{c}\text { No }(\%) \\
6619(64.75 \%)\end{array}$ & & $\begin{array}{c}\text { Yes }(\%) \\
4643(45.42 \%)\end{array}$ & $\begin{array}{c}\text { No }(\%) \\
5580(54.58 \%)\end{array}$ & \\
\hline \multicolumn{7}{|l|}{ Age of women } \\
\hline $15-24$ & $845(36.8 \%)$ & $1453(63.2 \%)$ & $<0.001$ & $1097(47.8 \%)$ & $1200(52.2 \%)$ & $<0.001$ \\
\hline $25-34$ & $1721(38.7 \%)$ & $2731(61.3 \%)$ & & $2058(46.2 \%)$ & $2393(53.8 \%)$ & \\
\hline $35-49$ & $1039(29.9 \%)$ & $2436(70.1 \%)$ & & $1488(42.8 \%)$ & $1987(57.2 \%)$ & \\
\hline \multicolumn{7}{|c|}{ Educational level } \\
\hline No education & $1930(30.9 \%)$ & $4324(69.1 \%)$ & $<0.001$ & $2319(37.1 \%)$ & $3934(62.9 \%)$ & $<0.001$ \\
\hline Primary & $1130(39.0 \%)$ & $1766(61.0 \%)$ & & $1521(52.5 \%)$ & $1374(47.5 \%)$ & \\
\hline Secondary & $331(50.6 \%)$ & $323(49.4 \%)$ & & $465(71.1 \%)$ & $189(28.9 \%)$ & \\
\hline Higher & $213(50.6 \%)$ & $208(49.4 \%)$ & & $338(80.5 \%)$ & $82(19.5 \%)$ & \\
\hline \multicolumn{7}{|l|}{ Wealth index } \\
\hline Poorest & $380(19.5 \%)$ & $1573(80.5 \%)$ & $<0.001$ & $646(33.1 \%)$ & $1307(66.9 \%)$ & $<0.001$ \\
\hline Poorer & $643(31.0 \%)$ & $1431(69.0 \%)$ & & $841(40.5 \%)$ & $1233(59.5 \%)$ & \\
\hline Middle & $755(36.7 \%)$ & $1301(63.3 \%)$ & & $854(41.5 \%)$ & $1203(58.5 \%)$ & \\
\hline Richer & $811(40.6 \%)$ & $1189(59.4 \%)$ & & $886(44.3 \%)$ & $1114(55.7 \%)$ & \\
\hline
\end{tabular}


Visited health facility

$\begin{array}{lllllll}\text { No } & 1583(30.3 \%) & 3641(69.7 \%) & <0.001 & 2028(38.8 \%) & 3196(61.2 \%) & <0.001 \\ \text { Yes } & 2021(40.4 \%) & 2978(59.6 \%) & & 2615(52.3 \%) & 2384(47.7 \%) & \end{array}$

Distance to HF

Big problem

Not a big problem

$1659(29.7 \%)$

$3923(70.3 \%)<0.001$

$2142(38.4 \%)$

$3440(61.6 \%)<0.001$

$1945(41.9 \%) \quad 2696(58.1 \%)$

$2501(53.9 \%) \quad 2140(46.1 \%)$

Media Exposure

No

Yes

$1973(31.3 \%)$

$4336(68.7 \%)$

$<0.001$

2393(37.9\%)

$2250(57.5 \%)$

$3916(62.1 \%)<0.001$

1631(41.7\%)

$2284(58.3 \%)$

$1664(42.5 \%)$

\section{Religion of women}

Orthodox

Catholic

Protestant

Muslim

Other

Place of residence

Urban

Rural

Contextual regions

$$
\text { City }
$$

Agrarian

Pastoralist
$1839(44.4 \%)$

$34(45.3 \%)$

956(41.8\%)

$754(21.3 \%)$

21(11.7\%)

2301(55.6\%)

$41(54.7 \%)$

1333(58.2\%)

$2786(78.7 \%)$

$158(88.3 \%)$

$<0.001$

$2152(52.0 \%)$

$31(41.3 \%)$

1005(43.9\%)

1386(39.2\%)

69(38.5\%)

$1987(48.0 \%)<0.001$

$44(58.7 \%)$

1284(56.1\%)

2154(60.8\%)

$110(61.5 \%)$ 
modern contraceptive methods of the same clusters was $0.5851\left(=e^{-0.5359}\right)$ times the estimated odds of women living in urban areas of the same clusters keeping all other variables constant. This shows women living in the urban area of the same clusters are more likely to use the modern contraceptive method than women living in rural areas. The odds of women in age 35-49 years using modern contraceptive methods of the same clusters was $0.6228\left(=e^{-0.4734}\right)$ times estimated odds of women in age 15-24 years of the same clusters keeping all other variables constant. The odds of women visited a health facility in the last 12 months are using modern contraceptive methods was $2.0177\left(=e^{0.702}\right)$ times the odds of women not visited a health facility in the last 12 months of the same clusters of women not visited a health facility in the last 12 months of the same cluster keeping all other variables constant. A woman in the poorest wealth index was less likely to use the modern contraceptive method as compared to the middle wealth index. Being a follower of the Muslim religious decreases the likelihood of using modern contraceptive methods by $54.62 \%$.

Table 3: The Goodness of fit statistics of bivariate multilevel logistics regression on modern contraceptive method and knowledge of HIV prevention with considering cluster-specific random effects.

\begin{tabular}{cccc}
\hline \multicolumn{2}{c}{ Knowledge of HIV prevention } & \multicolumn{2}{c}{ Modern contraceptive use } \\
\hline$-2 \log$ L(HIVpk | r. effects) & 11781.09 & $-2 \log$ L(ModernCU | r. effects) & 10785.08 \\
Pearson Chi-Square & 9556.4 & Pearson Chi-Square & 9167.1 \\
Pearson Chi-Square / DF & 0.97 & Pearson Chi-Square / DF & 0.93 \\
\hline
\end{tabular}


Table 4: The result from bivariate multilevel logistics regression on modern contraceptive method and knowledge of HIV prevention by socio-economic and demographic predictor of women reproductive age across the clusters (EDHS 2016).

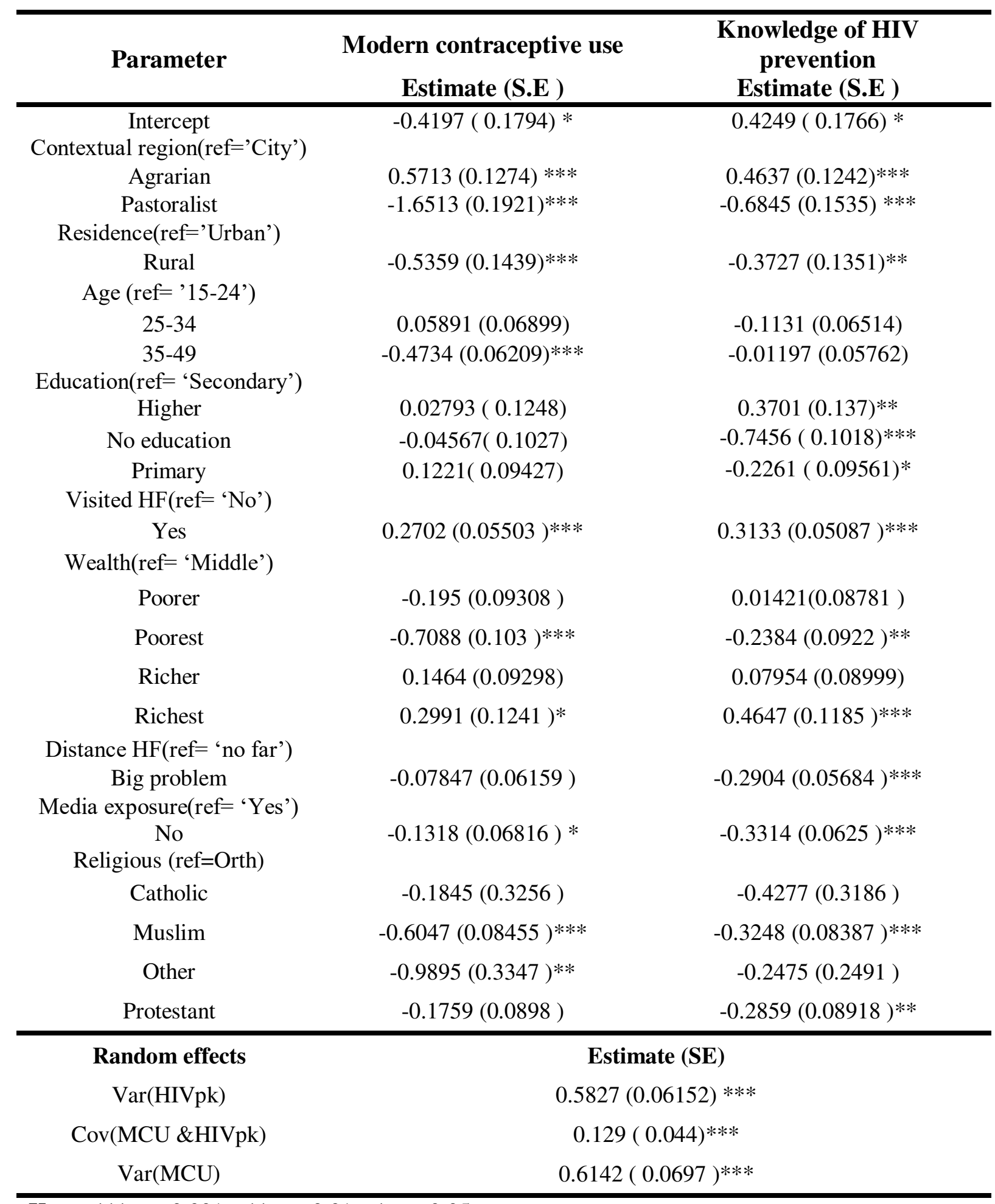

Key: $* * * \mathrm{p}<0.001, * * \mathrm{p}<0.01, * \mathrm{p}<0.05$ 
The odds of women living in pastoralist knew about HIV prevention of the same clusters was $0.5043\left(=e^{-0.6845}\right)$ times the estimated odds of women living in city contextual region of the same clusters keeping all other variables constant. The odds of women living in rural area knew about HIV prevention of the same clusters was $0.6889\left(=e^{-0.3727}\right)$ times the estimated odds of women living in urban areas of the same clusters keeping all other variables constant. The odds of women in the highest educational level knew about HIV prevention of the same clusters was $1.4478(=$ $e^{0.3701}$ ) times the estimated odds of women in the secondary educational level of the same clusters knew about HIV prevention was higher by $44.78 \%$ of estimated odds of women in the secondary educational level of the same clusters keeping all other variables constant. On the contrary, odds of women at no educational level (illiterates) knew about HIV prevention was lower by $47.44 \%$ of the estimated odds of women in the secondary educational level of the same clusters keeping all other variable constant. The odds of women visited a health facility in the last 12 months knew about HIV prevention of the same clusters was $1.3679\left(=e^{0.3133}\right)$ times the estimated odds of women not visited a health facility in the last 12 months of the same clusters keeping all other variables constant. A woman with the richest wealth index was more likely to know about HIV prevention as compared to the middle wealth index category. A woman having a big problem in distance from a health facility was less likely to know about HIV prevention as compared to women not facing a big problem distance from a health facility. Women not exposed to media were less likely to know about HIV prevention as compared to those women's exposure to media.

\section{Discussion}

This study was identified the joint predictors of modern contraceptive use and knowledge of HIV prevention of reproductive age women in Ethiopia with taking into account the association of responses in the same clusters, using 2016 Ethiopian Demographic and Health Survey data. The bivariate multilevel logistics regression model found that visiting a health facility in the last 12 months, wealth index, media exposure, religion, place of residence, and contextual region were predictors of nonusers of modern contraceptive and unawareness of HIV/AIDS prevention among women of reproductive age across the clusters in Ethiopia. The random-effects model estimated that the positive association between modern contraceptive use and knowledge of HIV prevention among women of reproductive age across the cluster, which was the indicators of the bivariate 
model have the best performance over the separate models, this finding was consistent with the study (Pierewan and Tampubolon, 2015; Han, 2016).

This study revealed that the use of the modern contraceptive methods was varied among a woman across the clusters, this finding consistent with the study (Khan, 1997). Place of residence and the contextual region was an important predictor of modern contraceptives use. The woman living in the urban area were more likely to use modern contraceptive methods as compared to the woman lives in rural areas in the same clusters and this finding was consistent with another study (Bogale et al., 2015). The woman who visited a health facility in the last 12 months were more likely to use a modern contraceptive method and this finding was consistent with the study (Antenane, 2002). Regarding the wealth index, this study demonstrated that women with the poorest wealth index were less likely to use modern contraceptive methods than women with the middle wealth index category in the same clusters. Moreover, a woman with the richest wealth index was more likely to use a modern contraceptive method as compared to the woman with the middle wealth index in the same clusters and this finding was consistent with another study (Clara Ladi, Tukur, and Alhaji, 2015; Alemayehu et al., 2016; Babiarz et al., 2017).

This study shows that the exposures to social media have advantages in promoting the advantages in the use of modern contraceptive methods and the woman exposed to media were more likely to use modern contraceptive methods as compared to women not exposed to media in the same clusters and this finding was consistent with the study (Khan, 1997; Kulkarni, 2003; Khan and Shaw, 2011). The religious belief of the woman decreases the likelihood of using modern contraceptive methods and this finding was consistent with the study (Alemayehu et al., 2016).

This study revealed that the woman's knowledge of HIV prevention was varied across the clusters and this finding was consistent with the study (Igulot and A.Magadi, 2018; Kumar and Mehendale, 2019). The woman living in the rural area and pastoralist contextual region was less likely to know about HIV prevention as compared to the woman who lives in an urban area and city contextual region, consistent with the finding of the study(Zhang et al., 2019). Woman having a higher educational level was more likely to know about HIV prevention as compared to women with no education and this finding were consistent with the study(Kyoga et al., 2013; Zhang et al., 2019). 
A woman with the richest wealth index was high knowledge of HIV prevention as compared to a woman with a middle wealth index (Bignami et al., 2007; Igulot and A.Magadi, 2018). The distance to the health facility was an impact on knowledge of HIV/AIDS prevention. Meaning, as a woman experiencing a big problem to health facility they less likely to know about HIV prevention and consistent with the study (Kyoga et al., 2013). Exposure to social media was a significant implication on the knowledge of HIV prevention. As women were more exposed to social media, the likelihood of knowing about HIV prevention was high and this finding was consistent with the study (Kyoga et al., 2013; Zhang et al., 2019).

As the limitation of this study, future studies should look for the community-level cultural beliefs and availability of a health facility to explore the variation in the geographical social difference on using modern contraceptive methods and knowledge of HIV prevention of women.

\section{Conclusion}

According to the findings of this study positive association between modern contraceptive use and knowledge of HIV prevention was found and women who use modern contraceptive methods were more likely to know about HIV prevention and vice versa. The variation that has been observed in the use of modern contraceptive methods and knowledge of HIV prevention in women across clusters was jointly significantly associated with place of residence, the contextual region of women at community level and wealth index, media exposure, visited heath facility, and religious belief at individual levels.

The use of modern contraceptive methods has a spillover effect on knowledge of HIV prevention of women across the clusters, which seemed to be the influence of education was contributed to one's use of the modern contraceptive method and a positive association between the use of modern contraceptive methods and knowledge of HIV prevention among the woman was found.

So, the integration of the two programs with their common risk factors can have advantages in terms of clinical benefit, cost, and care to seek individuals. Therefore, the intervention measures should be undertaken by the concerned body at the community level to enhance the use of modern contraceptive methods and knowledge of HIV prevention of women across the clusters. 


\section{List of abbreviations}

AIC: Akaike information criteria; BIC: Bayesian information criteria; HIV: Human Immunodeficiency Virus; AIDS: Acquired Immunodeficiency syndrome; EDHS: Ethiopian demographics and health survey; GLMM: Generalized linear mixed model; WHO: World Health Organization; ICC: Intraclass correlation coefficient; CSA: Central Statistical Agency; SAS: Statistical Analysis Software; EA: Enumeration area; STD: Sexually Transmitted Disease; PHC: Population Housing Census.

\section{Declarations}

\section{Ethics approval and consent to participate}

Ethiopian DHS was obtained ethical clearance from the Ethiopian Health Nutrition and Research Institute (EHNRI) Review Board, the national research Ethics Review committee (NRERC) at the Ministry of Science and Technology of Ethiopia, the Institute Review Board (IRB) of ICF international, and the Centre for Disease Control (CDC) and where the participant is children (under 16 years old), written informed consent for participation in the study was obtained from their guardian or parent. A permission letter to download the 2016 EDHS data set was also obtained from the DHS program after requesting ( $\underline{\text { www.measuredhs.com) }}$ website. The requested data were treated strictly confidential and were used only for this study.

\section{Consent for publication}

'Not applicable'

\section{Availability of data and materials}

All datasets used during this study are available at (www.measuredhs.com) website.

\section{Competing interests}

The authors declared that they have no competing interests.

\section{Funding}


The authors received no specific funding for this study.

\section{Author's contributions}

DB involved in the design and conception of the study, the analysis, interpretation of the findings, and write up the manuscript. EK approved the research proposal with some revision and comments by critically reviewing the manuscript as the role of the supervisor. All the authors approved the final version of the manuscripts.

\section{Acknowledgments}

We would like to appreciate the measure DHS program for providing us the data to conduct this study.

\section{Authors' information}

1*Departments of Statistics, College of Natural and Computational Sciences, Dire Dawa University, Dire Dawa, Ethiopia.

${ }^{2}$ Departments of Statistics, College of Science, Bahir Dar University, Bahir Dar, Ethiopia

\section{Reference}

Alemayehu, M. et al. (2016) 'Family planning use and associated factors among pastoralist community of afar region, eastern Ethiopia', BMC Women's Health, pp. 1-9. doi: 10.1186/s12905-016-0321-7.

Antenane, K. (2002) 'Attitudes Toward Family Planning and Reasons for Nonuse among Women with Unmet Need for Family Planning in Ethiopia', (Calverton, Maryland USA: ORC Macro.).

Babiarz, K. S. et al. (2017) 'Family Planning and Women's Economic Empowerment : Incentive Malaysian Women Working Paper 471 December 2017', (December 2017).

Bignami, S. et al. (2007) 'DHS WORKING PAPERS A study of the association of HIV infection with wealth in sub-Saharan Africa', (DHS working papers).

Bogale, B. et al. (2015) 'Married women' s decision making power on modern contraceptive use in urban and rural southern Ethiopia', pp. 5-11.

Breslow, N. E. and Clayton, D. G. (1993) 'Approximate Inference in Generalized Linear Mixed Models', Journal of the American Statistical Association, 88(421), p. 9. doi: 10.2307/2290687. 
Center, K. E. et al. (2016) 'Contraceptive use and uptake of HIV-Testing among sub-saharan african women', PLoS ONE, 11(4), pp. 1-11. doi: 10.1371/journal.pone.0154213.

Clara Ladi, E., Tukur, D. and Alhaji, A. A. (2015) 'Contextual Factors Influencing Modern Contraceptive Use in Nigeria', 120(Rockville, Maryland, USA: ICF International.DHS working paper).

Damon, M. B. and Robert, C. (2015) Multivariate Generalized Linear Mixed Models Using R, A CHAPMAN \& HALL BOOK. doi: 10.1017/CBO9781107415324.004.

Darroch, J. E., Sedgh, G. and Ball, H. (2011) 'Contraceptive Technologies : Responding to Women's Needs', Guttmacher Institute, (April), pp. 1-51.

Federal HIV/AIDS Prevention and Control Office (2020) 'HIV Prevention in Ethiopia National Road Map', (November 2018).

Gebre, M. N. and Edossa, Z. K. (2020) 'Modern contraceptive utilization and associated factors among reproductive-age women in Ethiopia: Evidence from 2016 Ethiopia demographic and health survey', BMC Women's Health, 20(1), pp. 1-14. doi: 10.1186/s12905-020-00923-9.

Girum, T. et al. (2018) 'Gender disparity in epidemiological trend of HIV / AIDS infection and treatment in Ethiopia', pp. 1-10.

Han, S. (2016) 'Applications of Business Analytics in Marketing : Joint Modeling of Correlated Multivariate Outcomes', University of Tennessee, Knoxville Trace: Tennessee Research and Creative Exchange, Dissertati.

Hope, R. et al. (2014) 'Health Systems Integration of Sexual and Reproductive Health and HIV Services in Sub-Saharan Africa : A Scoping Study’, 67, pp. 259-270.

Hox, J. J. (2010) 'Multilevel Analysis : Techniques and Applications', in Quantitative methodology series. Third edit.

Hrusa, G. et al. (2020) 'Quality of family planning counseling in Ethiopia: Trends and determinants of information received by female modern contraceptive users, evidence from national survey data, (2014- 2018)', PLoS ONE, 15(2), pp. 1-18. doi:

10.1371/journal.pone.0228714.

ICF, C. S. A. (CSA) [Ethiopia] (2016) Demographic and Health Ethiopia 2016.

Igulot, P. and A.Magadi, M. (2018) 'Socioeconomic Status and Vulnerability to HIV Infection in Uganda : Evidence from Multilevel Modelling of', 2018.

Institute, T. E. P. H. (2017) 'HIV Related Estimates and Projections for Ethiopia - 2017 March 2017', (March).

Khan, H. R. and Shaw, J. E. H. (2011) 'Multilevel logistic regression analysis applied to binary contraceptive prevalence data', Journal of Data Science, 9, pp. 93-110. 
Khan, H. T. A. (1997) 'A hierarchical model of contraceptive use in urban and rural Bangladesh', Contraception, 55(2), pp. 91-96. doi: 10.1016/S0010-7824(96)00278-8.

Kulkarni (2003) 'Health and Population -', 26(2), pp. 87-93.

Kumar, R. J. and Mehendale, S. M. (2019) 'Determinants of consistently high HIV prevalence in Indian Districts : A multi-level analysis', pp. 1-14.

Kyoga, L. et al. (2013) 'Comprehensive knowledge of HIV prevention among fishing communities of’, (2020), pp. 1-8.

LEONARDO, G. et al. (2016) 'Exploiting timss and pirls combined data: multivariate multilevel modelling of student achievement', The Annals of Applied Statistics, 10(4), pp. 2405-2426. doi: 10.1214/16-AOAS988.

Nations, U. (2019) 'World Population Prospects 2019’.

Ousman, S. K., Magnus, J. H. and Sundby, J. (2020) 'Uptake of Skilled Maternal Healthcare in Ethiopia : A Positive Deviance Approach', pp. 7-9.

Pierewan, A. C. and Tampubolon, G. (2015) 'Happiness and Health in Europe : A Multivariate Multilevel Model', pp. 237-252. doi: 10.1007/s11482-014-9309-3.

Report, W. D. (2019) WORLD DEVELOPMENT REPORT. doi: 10.1596/978-1-4648-1328-3.

Schabenberger, O. (2005) 'Introducing the GLIMMIX procedure for Generalized Linear Mixed Models', pp. 1-20.

Wang, J. (2013) 'Joint Modeling of Binary Response and Survival Data in Clinical Trials By'.

WHO, UNICEF, UNFPA, W. B. G. and the U. N. P. D. . (2019) Maternal mortality: level and trends 2000 to 2017, Sexual and Reproductive Health. Available at:

https://www.who.int/reproductivehealth/publications/maternal-mortality-2000-2017/en/.

Yu, X. et al. (2018) 'Computation Joint models of dynamics of mothers' stress and children ' $\mathrm{s}$ disease', Communications in Statistics - Simulation and Computation, 0(0), pp. 1-16. doi: 10.1080/03610918.2018.1468455.

Zhang, T. et al. (2019) 'Awareness of HIV / AIDS and its routes of transmission as well as access to health knowledge among rural residents in Western China : a cross-sectional study', pp. $1-11$. 
Figures

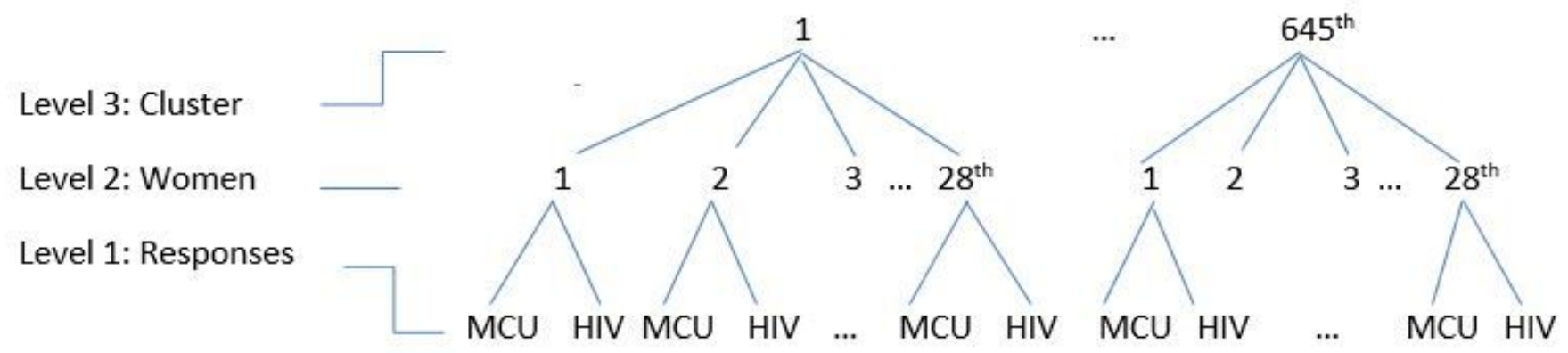
where MCU is modern contraceptive use. HIV is knowledge of HIV.

Figure 1

Hierarchal structures of the data 